\title{
Apoio social: percepção materna em contextos com diferentes graus de urbanização
}

\author{
Viviane Vieira \\ Mauro Luis Vieira \\ Universidade Federal de Santa Catarina \\ Alessandra Bonassoli Prado \\ Universidade de São Paulo
}

\begin{abstract}
Resumo
Esta pesquisa tem como objetivos: verificar as diferenças no apoio percebido por mães de três contextos de urbanização; analisar a correlação do apoio com dados sociodemográficos e com a qualidade do ambiente familiar; e estabelecer qual modelo de variáveis explica o apoio percebido em cada contexto. Ao total, 150 mães residentes em três contextos responderam um questionário sobre dados sociodemográficos, itens da qualidade de vida familiar atual e uma Escala de Apoio Social. Por meio de análises estatísticas, constataram-se diferenças significativas nas dimensões do apoio social, com melhor percepção nas dimensões Apoio Afetivo e Apoio Emocional para o Interior Leste e menor percepção do Apoio Material para a Capital. Nos modelos de regressão, as variáveis número de filhos e qualidade do ambiente familiar atual mostraram-se preditoras do apoio. Conclui-se que a qualidade do ambiente familiar tem importância no apoio e as características individuais e culturais devem ser consideradas ao se investigar o suporte social.
\end{abstract}

Palavras-chave: apoio social; relações familiares; urbanização.

\begin{abstract}
The relationship between cultural models, social support and quality of family environment in context with different degrees of urbanization. The present study aimed to investigate the differences in perceived social support for mothers of three contexts with distinct urbanization; to analyze the correlation of support with the quality of the family environment and to establish which variables models explain the perceived support in each context. A total of 150 mothers living in three contexts answered a questionnaire about sociodemographic data, items of current quality of family life and Scale of Social Support. Through the statistical analysis it has been found significant differences in dimensions of Social Support, with best perception of the Affective and Emotional support in Eastern context and lower perception for material support in the Capital. In regression models, the number of children and current quality of family life proved to be the predictors of support. It has been concluded that the quality of family environment is important in supporting and both individual characteristics and cultural characteristics should be considered when investigating social support.
\end{abstract}

Keywords: social support; family relations; urbanization.

A rede de apoio ou suporte social diz respeito àquelas interações que se tornam significativas e produzem efeitos benéficos para os indivíduos (Crittenden,1985; Koeske \& Koeske, 1990; Nelson, 2004). Essa rede, segundo Berkman (1984), tornou-se valorizada como objeto de estudos a partir da década de 70 , especialmente pela descoberta da importância e vantagem que o suporte social traz para o indivíduo, principalmente em relação à saúde de modo geral. Autores apontam que a rede de apoio eficiente é um fator de proteção contra o estresse, mortalidade e morbidades (Andrade \& Vaitsman, 2002; Castro, Campero, \& Hernandez, 1997; Cutrona,
Cole, Colangelo, Assouline, \& Russel, 1994; Matsukura, Marturano, \& Oishi, 2002; Montigny, Lacharité, \& Amyot, 2006). Além disso, Azevedo e Arrais, (2006) indicam que a saúde mental também sofre influência do apoio, indicando que esse suporte está ligado com a diminuição de distúrbios psicológicos como a depressão.

Contudo, os benefícios da rede social só ocorrem quando as pessoas pertencentes a ela percebem esse apoio, pois a rede de apoio social - compreendida como um sistema composto por várias pessoas, funções e situações que ofereçam apoio instrumental, material e emocional à pessoa em diferentes 
necessidades (Berkman, 1984; Dessen \& Braz, 2000; Nelson, 2004; Vanderlinde, Borba, \& Vieira, 2009) - nem sempre está disponível em todas as situações. Dependendo do momento, somente uma pessoa (ou poucas pessoas) pertencente à rede social da pessoa fornecerá um suporte ou apoio efetivo (Crittenden, 1985).

Assim, conceitualmente, o suporte ou apoio social pode ser entendido como o conjunto de recursos postos à disposição por outras pessoas que possam oferecer ajuda, encorajamento e conselhos que levam o indivíduo a sentir-se ou acreditar ser cuidado, amado, seguro e pertencente a um grupo (Berkman, 1984; Castro et al., 1997; Goodwin, Cost, \& Adonu, 2004; Koeske \& Koeske, 1990; Nelson, 2004). O apoio social também envolve a satisfação do indivíduo com o apoio recebido, ou seja, como ele está percebendo o suporte oferecido a ele (Crittenden, 1985).

Sarason, Levine, Basham e Sarason (1983) apontam que, por um tempo, o apoio percebido foi pouco investigado pelas pesquisas nessa área, pois se acreditava que o número de pessoas e situações concretas em que o apoio ocorria era mais importante que a inferência do sujeito que recebia esse apoio. Entretanto, com a descoberta da importância da satisfação do indivíduo com a rede de apoio como primordial à sensação de sentir-se amado e seguro, a percepção do sujeito sobre o suporte recebido tornou-se essencial às pesquisas (Berkman, 1984; Montigny et al., 2006; Nelson, 2004).

Uma das maiores influências benéficas da rede de apoio social efetiva é seu efeito atenuante sobre o estresse. Segundo a literatura sobre o assunto, uma rede de apoio social efetivo ajuda o indivíduo a enfrentar momentos difíceis e a lidar com eventos estressantes, melhorando as habilidades adaptativas do sujeito diante de novas situações (Castro et al., 1997; Goodwin et al., 2004; Montigny et al., 2006; Nelson, 2004; Sarason et al., 1983).

Um dos momentos do ciclo vital da família que representa intenso estresse na vida de um indivíduo é o início da parentalidade, quando os novos pais precisam aprender como lidar com a nova criança e enfrentam vários desafios, necessitando de diversas fontes de suporte, tanto material quanto emocional, para lidarem com as novas situações. A parentalidade exige dos cuidadores flexibilidade e segurança para lidar com seus filhos e o apoio social recebido pelas mães tem sido apontado como um fenômeno que pode influenciar de modo positivo na relação entre mãe e filho (Bornstein, Putnick, Suwalsky, \& Gini, 2006; Jennings, Stagg, \& Connors, 1991; Leinonen et al., 2003; Montigny et al., 2006). As principais figuras de apoio são o parceiro amoroso e pai da criança, os avós, os amigos, parentes próximos e mesmo os profissionais de saúde (Crittenden, 1985; Cutrona et al., 1994; Dessen \& Braz, 2000; Montigny et al., 2006; Saranson et al., 1983; Vanderlinde et al., 2009).

Os estudos relacionados com o apoio social também investigam outras variáveis além da parentalidade, como as características sociodemográficas envolvidas na redução ou aumento da percepção do apoio social. A renda foi a variável mais investigada, sendo verificado que está relacionada positivamente com o apoio, ou seja, quanto maior a renda, mais o apoio apontado (Castro et al., 1997; Hashima \& Amato, 1994;
Leinonem, Solantus, \& Punamäki, 2003). Da mesma forma, a escolaridade e a idade se relacionam positivamente com apoio (Crittenden, 1985; Griep, Chor, Faerstein, Werneck, \& Lopes, 2005).

Além dos fatores apontados anteriormente, o contexto cultural também deve ser considerado para se compreender de modo mais holístico o apoio social. Características da população local, modo de se relacionar com o grupo social e valorização dos aspectos sociais ou individuais, devem ser considerados ao se investigar o apoio social. É importante verificar que contextos mais urbanizados apresentam características mais individualistas, enquanto locais com menores níveis de urbanização tenderiam a ter uma maior proximidade com o grupo social. A forma que o grupo interatua e as suas relações podem refletir nos cuidados parentais (Keller et al., 2004; Keller, Borke, \& Yovsi, 2005; Westphal, Vieira, Vieira, \& Prado, 2010) e, consequentemente, na forma como esses pais percebem o suporte durante os cuidados com o filho.

Baseando-se na introdução teórica apresentada, esse estudo analisa como mães catarinenses percebem o apoio social recebido, tendo como objetivos: a) verificar as diferenças e similaridades no apoio percebido entre três contextos com diferentes graus de urbanização; b) analisar quais dados sociodemográficos estão correlacionados com o apoio em cada contexto; e c) estabelecer qual modelo de variáveis explica o apoio percebido pelos participantes em todos os contextos. Além disso, busca-se, também, correlacionar a escala de apoio social percebido com qualidade do ambiente familiar atual e verificar como esta variável se relaciona com o apoio.

Espera-se encontrar distinções nos contextos estudados com relação às dimensões do apoio social, de modo que nos locais menos urbanizados e interdependentes o apoio será mais percebido, especialmente na dimensão material. Não obstante, espera-se que semelhanças na percepção do apoio entre contextos de proximidade cultural também ocorram. Ainda com relação ao apoio, tem-se como hipótese que as variáveis renda, escolaridade e a qualidade do ambiente familiar atual serão preditores da percepção desse suporte social nos contextos estudados.

\section{Método}

\section{Participantes $^{l}$}

No total, participaram 150 mães, sendo 50 residentes em cada um dos três contextos distintos, conforme será descrito no próximo parágrafo. As participantes deveriam ter a partir de 20 anos de idade, com pelo menos um filho com idade entre zero e seis anos de idade. Os três contextos, nos quais foram realizadas as coletas de dados, receberam a classificação de Capital, Interior Leste e Interior Oeste, todos situados no Estado de Santa Catarina.

A amostra do contexto chamado Capital foi composta por mães com idade entre 21 e 49 anos $(M=32,14 ; D P=6,37)$, número de filhos entre um e quatro, sendo que a maioria tinha um filho $(60 \%)$ no período da coleta de dados. A maior parte dessas mulheres era casada ou vivia em união estável (84\%) com composição familiar nuclear (74\%), era de religião católica 
(52\%), tinha trabalho remunerado $(78 \%)$, foi criada na zona urbana $(84 \%)$, tinha escolaridade superior $(72 \%)$ e possuía renda mensal familiar acima de R\$2.000,00 (70\%). Como capital de estado, o local é considerado como tendo alto nível de urbanização no tocante à infraestrutura (Instituto Brasileiro de Geografia e Estatística - IBGE, 2007).

No contexto Interior Leste, a amostra foi composta por mães residentes em duas cidades, com idade entre 21 e 38 anos $(M=$ $29,16 ; D P=3,82)$, total de filhos entre um e quatro, sendo que a maioria tinha um (56\%) ou dois filhos (34\%) no período da coleta de dados. Em sua maior parte essas mulheres eram casadas ou viviam em união estável (94\%) com composição familiar nuclear (88\%), eram católicas (66\%), foram criadas na zona rural (56\%), possuíam trabalho remunerado (84\%), escolaridade entre médio completo e superior incompleto (54\%) e possuíam renda mensal familiar entre $\mathrm{R} \$ 301,00$ e R \$ 1.300,00 (70\%). O agrupamento de duas cidades para fins de coleta de dados justifica-se por estas serem semelhantes em vários aspectos. A primeira cidade é de colonização alemã, italiana e polonesa e possui população estimada de 12.758 habitantes em 2007 (IBGE, 2007). A segunda cidade também é de colonização alemã e tem 12.776 habitantes (IBGE, 2007). Em termos de infraestrutura, consideram-se essas cidades como tendo nível de urbanização em desenvolvimento, sendo que há uma proximidade territorial destas com os pólos da indústria metal-mecânica.

No contexto Interior Oeste, a amostra foi composta por mães com idade entre 20 e 43 anos $(M=29,80 ; D P=6,06)$, total de filhos entre um e cinco, sendo que a maioria tinha um $(30 \%)$ ou dois filhos $(42 \%)$ no período da coleta de dados. Em sua maioria essas mulheres eram casadas ou viviam em união estável (94\%) com composição familiar nuclear (82\%)m católicas $(88 \%)$, não trabalhavam $(66 \%)$, foram criadas na zona rural (68\%), tinham escolaridade até ensino fundamental completo (64\%) e possuíam renda mensal familiar de até R $\$ 1.000,00$ (68\%). Este contexto situa-se no extremo oeste do estado e é de colonização alemã e italiana. Possui 10.604 habitantes e seu nível de desenvolvimento é considerado baixo, predominando as áreas destinadas a lavouras e pastagens (Instituto Brasileiro de Geografia e Estatística, 2007).

\section{Instrumento}

Esse estudo é parte de uma pesquisa maior que continha um instrumento com diversas escalas. Para esse artigo somente três partes do instrumento foram investigadas:

Caracterização sociodemográfica. Inclui escolaridade da família, renda familiar mensal, número de pessoas na casa, número de filhos, número de cômodos na casa e idade da mãe no nascimento do primeiro filho.

Qualidade do ambiente familiar atual. Perguntas sobre o clima familiar, relação do casal e afetividade. As respostas eram dadas por meio de uma escala de cinco pontos com denominações diferentes para cada questão. A partir da junção de seis itens sobre a qualidade de vida familiar atual (clima familiar, conflitos com o parceiro, relação do casal e afeto parental), foi construída a variável Qualidade do ambiente familiar.
Escala de Aferição de Apoio Social. Originalmente elaborada por Sherbourne e Stewart (1991), foi traduzida, adaptada e validada para o Brasil pelo Estudo Pró-Saúde (Griep et al., 2005). Trata-se de uma escala tipo Likert com cinco pontos ( 1 = "nunca" até $5=$ "sempre"), que avalia a frequência com que a mãe percebe seus laços sociais e recursos de outras pessoas de sua rede em caso de necessidade. Esta versão da escala mantém o conjunto original de 19 itens e inclui três fatores: Apoio Material (por exemplo: 'você conta com alguém que a ajude, se ficar de cama'), Apoio Emocional/Informação (ex.: 'há alguém para dar bons conselhos em situação de crise') e Interação Social Positiva/Apoio Afetivo (ex.: 'você tem alguém com quem fazer coisas agradáveis').

\section{Procedimento}

O contato inicial com as mães foi feito por meio de creches, centros de educação infantil e postos de saúde. Além disso, cada participante indicava outras para a pesquisa.

As entrevistas, com duração média de uma hora, eram agendadas em contato prévio com as mulheres, que escolhiam o local, a data e o horário de sua preferência. Ficavam presentes nas entrevistas a aplicadora, a entrevistada e, ocasionalmente, a criança (caso a mãe assim desejasse). A participante recebia um modelo do questionário para acompanhamento enquanto a aplicadora realizava as perguntas.

A pesquisa teve início após a aprovação pelo Comitê de Ética da Universidade Federal de Santa Catarina (números 166/06, para o Interior, e 239/06, para a Capital); todas as participantes assinaram o Termo de Consentimento Livre e Esclarecido.

\section{Análise de dados}

Inicialmente, os dados obtidos nos três contextos foram analisados comparando-se as médias por meio da análise de variância (ANOVA) para medidas repetidas e o teste post hoc de Tukey, para verificar em que comparações (dois-a-dois) se localizam essas diferenças (Dancey \& Reidy, 2006).

Os três contextos desse estudo também foram analisados separadamente, por meio de análise de correlações, na qual o teste $r$ de Pearson foi utilizado para mostrar o grau de relacionamento entre as variáveis e a probabilidade dessas relações ocorrerem por erro amostral (Dancey \& Reidy, 2006). As variáveis que demonstraram maior correlação foram selecionadas para a análise de regressão múltipla, método Stepwise, por meio do qual as variáveis selecionadas foram agrupadas e foram investigados os modelos que melhor explicam os principais fatores contribuintes para cada dimensão do apoio dentro dos contextos verificados.

\section{Resultados}

Como as análises realizadas foram feitas nos três contextos estudados, tanto de forma unificada quanto separada, optou-se por apresentar os resultados em quatro tópicos, definidos em função dos locais investigados: os três contextos unificados; contexto Capital; contexto Interior Leste; contexto Interior Oeste.

\section{a) Análises com os três contextos unificados}


Foram realizadas análises da escala de apoio social unificando os três contextos catarinenses estudados. As médias de cada contexto em cada dimensão da escala podem ser visualizadas na Tabela 1 .

As médias totais da escala e de cada dimensão foram comparadas por meio da análise de variância ANOVA para medidas repetidas. Nessa comparação das médias totais, a diferença foi estatisticamente significativa $(F=5,58 ; p<0,05)$. Essa análise apresentou o contexto Interior Leste com uma média significativamente maior nas dimensões Apoio Afetivo/ Interação Positiva $(D H S=0,24 ; p<0,05)$ e Apoio Emocional/Informação
$(D H S=0,42 ; p<0,05)$ em comparação com o contexto Interior Oeste. Nesta mesma análise, o contexto Capital aparece com médias significativamente menores que o contexto Interior Leste na dimensão Apoio Material $(D H S=0,62 ; p<0,05)$.

Após essas primeiras análises, foi realizado o teste $r$ de Pearson em que algumas variáveis sociodemográficas e as dimensões da escala de apoio social foram correlacionadas e verificado o relacionamento entre estas. As que apresentaram correlação foram para a análise de regressão múltipla para verificar o quanto essas variáveis explicam o apoio percebido pelas mães nos três contextos.

Tabela 1

Análise estatística das médias aritméticas das dimensões da Escala de Aferição de Apoio Social na comparação entre os três contextos

\begin{tabular}{|c|c|c|c|}
\hline $\begin{array}{l}\text { Dimensões de Apoio } \\
\text { Social }\end{array}$ & Contextos & Média & Desvio Padrão \\
\hline \multirow{4}{*}{ Afetivo/Interação Positiva } & Capital & 4,33 & $\pm 0,61$ \\
\hline & Interior Leste $^{\mathrm{a}}$ & 4,57 & $\pm 0,58$ \\
\hline & Interior Oeste $^{\mathrm{a}}$ & 4,12 & $\pm 0,88$ \\
\hline & $\mathrm{F}(5,59)=4,95^{*}$ & & \\
\hline \multirow{4}{*}{ Emocional/Informação } & Capital & 3,94 & $\pm 0,74$ \\
\hline & Interior Leste $^{\mathrm{a}}$ & 4,25 & $\pm 0,90$ \\
\hline & Interior Oeste $^{\mathrm{a}}$ & 3,83 & $\pm 0,83$ \\
\hline & $\mathrm{F}(5,59)=3,45^{*}$ & & \\
\hline \multirow{4}{*}{ Material } & Capital $^{\mathrm{a}}$ & 3,92 & $\pm 1,01$ \\
\hline & Interior Leste $^{\mathrm{a}}$ & 4,54 & $\pm 0,73$ \\
\hline & Interior Oeste & 4,13 & $\pm 0,95$ \\
\hline & $F(5,59)=6,03^{*}$ & & \\
\hline
\end{tabular}

As características sociodemográficas que se correlacionaram foram: qualidade do ambiente atual $(r=0,22 ; p<0,01)$; renda familiar $(r=0,23 ; p<0,01)$; número de filhos da respondente $(r=$ $0,19 ; p<0,05)$; escolaridade da respondente $(r=0,23 ; p<0,01)$ e o número de pessoas que moram na casa da respondente $(r=$ $0,19 ; p<0,05)$. Como o foco do estudo era no apoio social, essas variáveis correlacionadas foram consideradas independentes para realizar a análise de regressão e verificar um modelo de variáveis que expliquem o apoio. Os resultados indicaram que as variáveis "qualidade do ambiente familiar atual" e "renda familiar" explicam parcialmente o apoio percebido pelas participantes. Conjuntamente essas variáveis explicam $17 \%$ da variância, indicando que quanto melhor a qualidade do ambiente familiar atual e a renda, mais o apoio é percebido pelas respondentes.

Após a apresentação dos resultados que buscou comparar os três contextos conjuntamente, a próxima etapa das análises visa identificar as características de cada contexto separadamente, objetivando encontrar as similaridades e diferenças entre os locais de estudo e como o apoio é explicado em cada um destes.

\section{b) Contexto capital}

No contexto Capital a correlação de Pearson apontou algumas relações entre as variáveis analisadas. A variável "renda familiar mensal" apresentou correlação positiva com a "escolaridade" ( $r=0,78 ; p<0,01)$, indicando que quanto maior a renda declarada, maior a escolaridade. Além da correlação com a "renda familiar mensal", a "escolaridade" também demonstrou relações com todas as dimensões da escala de apoio $(p<0,01)$. A partir disso, pode-se compreender que quanto mais escolaridade a mãe apresentava, mais esta percebia o "Apoio Afetivo/Interação Positiva" ( $r=0,37)$, o "apoio emocional e de informação" ( $r=$ $0,48)$ e o "Apoio Material” ( $r=0,37)$.

Outras correlações com as dimensões da escala de apoio foram analisadas. O "Apoio Emocional e de Informação" apresentou correlação positiva $(p<0,05)$ com as variáveis "número de pessoas que moram na casa" $(r=0,30)$ e o "número de filhos" ( $r=0,29)$, indicando que quanto maior o número dessas variáveis, mais a mãe percebe o Apoio Emocional e de Informação. As outras duas dimensões do apoio demonstraram uma relação negativa com a variável "qualidade do ambiente familiar atual". Isso indica que quanto mais estressante o ambiente familiar menos a mãe percebeu o Apoio Afetivo/ Interação Positiva $(r=0,36 ; p<0,01)$ e o Apoio Material $(r$ $=0,35 ; p<0,05)$. A última correlação foi entre as dimensões do apoio. Essa demonstrou que quanto mais a mãe declarou uma das dimensões do apoio, mais as outras eram também percebidas.

A partir das correlações, as variáveis que apresentaram relação com as dimensões do apoio foram analisadas no método de regressão linear. Esta demonstrou três modelos. O 
primeiro, com a variável escolaridade da mãe $(\mathrm{E}=0,15 ; \beta=$ 0,$46 ; F=13,56 ; R^{2}$ ajustado $=0,20 ; p<0,01$ ), explica $20 \%$ da variância do apoio percebido. O segundo modelo inclui, além da "escolaridade" ( $\mathrm{E}=0,15 ; \beta=0,45 ; p<0,01)$, a variável qualidade do ambiente familiar atual $\left(\mathrm{E}=-0,22 ; \beta=-0,32 ; F=11,36 ; R^{2}\right.$ ajustado $=0,29 ; p<0,01)$, respondendo a $29 \%$ da variância do apoio social. O último modelo abrangeu a "escolaridade" $(\mathrm{E}=0,16 ; \beta=0,49)$, a "qualidade do ambiente familiar atual" $(\mathrm{E}=-0,18 ; \beta=-0,27 ; p<0,01)$ e o "número de filhos" ( $\mathrm{E}=$ 0,$23 ; \beta=0,24 ; F=9,42 ; R^{2}$ ajustado $\left.=0,34 ; p<0,001\right)$. Esses modelos indicam que a escolaridade mais elevada, a qualidade do ambiente familiar menos estressante e um maior número de filhos explicam 34\% da variância do apoio percebido.

\section{c) Contexto interior leste}

As correlações do contexto Interior Leste apontaram que, assim como a Capital, quanto maior a renda familiar mensal, maior a escolaridade da mãe $(r=0,29 ; p<0,05)$. A variável idade da respondente apresentou uma relação negativa com o "Apoio Material" $(r=0,30 ; p<0,05)$, compreendendo-se que quanto maior a idade da mãe, menos ela percebe o "Apoio Material". A última correlação encontrada demonstrou a mesma relação entre as dimensões da escala de apoio que o contexto analisado anteriormente, quanto mais cada dimensão é percebida, mas as outras também são apontadas pela mãe.

Para a análise de regressão múltipla, as variáveis "renda familiar mensal" e a "idade da respondente" foram colocadas como as variáveis independentes. Contudo, o método stepwise utilizado na regressão não apresentou nenhum modelo explicativo estatisticamente significativo.

\section{d) Contexto interior oeste}

O último contexto analisado, o Interior Oeste, indicou que, a exemplo de que foi constatado nos contextos anteriores, a "escolaridade" e a "renda familiar mensal" se correlacionam positivamente $(r=0,50 ; p<0,01)$. Essas duas variáveis também foram correlacionadas negativamente com a "qualidade do ambiente familiar atual" $(r=0,30 ; p<0,05)$. Já a correlação da "escolaridade" com o "Apoio Emocional/Interação Positiva" $(r$ $=0,30 ; p<0,05)$ indicou que quanto mais escolarizada a mãe era, mais ela percebia essa dimensão do apoio.

O "número de pessoas que moram na casa" demonstrou um relacionamento negativo com o "Apoio Afetivo/Interação Positiva" $(r=0,39 ; p<0,01)$ e com o "Apoio Emocional e de Informação" ( $r=0,34 ; p<0,01)$. Compreende-se assim, que os dados indicam que um maior número de pessoas na casa diminuiu a percepção da mãe com relação ao Apoio Afetivo e o Emocional. Assim como a variável anterior, o "número de filhos" correlacionou-se negativamente com as três dimensões da escala de apoio. Esse resultado aponta que quanto mais filhos a mãe tinha, menos ela percebia o "Apoio Afetivo/Interação Positiva" $(r=0,38 ; p<0,01)$, o "apoio emocional e de informação" $(r=$ $0,32 ; p<0,05)$ e o "Apoio Material" $(r=0,28 ; p<0,05)$.

A "qualidade do ambiente familiar atual" apontou correlação negativa com todas as dimensões do apoio. Isso indica que quanto mais tranquilo o ambiente familiar, mais a mulher percebe o "Apoio Afetivo/Interação Positiva" $(r=0,45 ; p<0,01)$, o "Apoio
Emocional e de Informação" $(r=0,40 ; p<0,01)$ e o "Apoio Material" $(r=0,36 ; p<0,01)$. Por fim, a análise apontou, da mesma forma que os contexto anteriores, que as dimensões da escala de apoio se correlacionam positivamente.

As variáveis independentes escolhidas a partir das correlações para a análise de regressão múltipla foram: "qualidade do ambiente familiar atual", "número de filhos", "renda familiar mensal" e "escolaridade da respondente". A partir disso, dois modelos estatisticamente significativos foram formulados.

O primeiro modelo indica a presença da variável "qualidade do ambiente familiar atual" ( $\mathrm{E}=-0,53 ; \beta=-0,48 ; F=14,42$; $R^{2}$ ajustado $\left.=0,21 ; p<0,001\right)$ explicando $29 \%$ da variância. $\mathrm{O}$ segundo modelo indica a "qualidade do ambiente familiar atual" ( $\mathrm{E}=-0,49 ; \beta=0,44 ; p<0,001)$ e o "número de filhos" $\left(\mathrm{E}=-0,23 ; \beta=0,33 ; F=12,08 ; R^{2}\right.$ ajustado $\left.=0,31 ; p<0,01\right)$ como dados que explicam $31 \%$ da variância do apoio percebido no contexto Interior Oeste. A partir disso, compreende-se que quanto mais tranquilo o ambiente familiar atual e menos filhos a mãe apresenta, mais o apoio é percebido pelas mesmas.

\section{Discussão}

A análise dos três contextos conjuntamente indicou que o Interior Leste foi o local com maiores médias em todas as dimensões da escala de apoio, incluindo uma diferença estatisticamente significativa com o Interior Oeste nas dimensões Apoio Afetivo/Interação Positiva e Apoio Emocional e de Informação. Outro estudo que analisou esses mesmos três contextos indicou que o Interior Leste apresenta características mistas de socialização com o grupo (proximidade social e incentivo do desenvolvimento individual, ou seja, independente), enquanto o Interior Oeste tem maior direcionamento para a interação com o grupo social em detrimento de dimensões individuais (interdependente) (Westphal et al., 2010).

A partir da análise entre esses dois contextos do Interior, pode-se destacar que, apesar do contexto Interior Oeste ter como característica um elevado grau de vinculação entre os membros do grupo social, isso não necessariamente significa a satisfação das mães com o apoio percebido. Sentir-se seguro e pertencente a um grupo é uma parte essencial para que os efeitos benéficos do apoio recebido sejam efetivos (Berkman, 1984; Montigny et al., 2006; Nelson, 2004; Sarason et al., 1983), e nem sempre essa percepção é garantida pelo número de pessoas ao redor.

A percepção do apoio social torna-se ainda mais subjetiva ao se examinarem as dimensões em que a diferença foi estatisticamente significativa. No caso do Apoio Afetivo/ Interação Positiva são situações que o sujeito percebe demonstrações físicas de amor e afeto e pessoas com quem contar para relaxar e se divertir; e no Apoio Emocional e de Informação é a percepção da rede social como satisfatória para as necessidades individuais em relação a problemas emocionais e poder contar com pessoas que aconselhem, informem e orientem (Griep et al., 2005). Extrai-se desses conceitos que essas dimensões envolvem mais questões da compreensão pessoal do sujeito diante de sua rede mais do que algo concreto.

Além da distinção entre as dimensões da escala, os dados 
sociodemográficos dos dois locais também apresentaram diferenciações, com o Interior Leste com maior escolaridade, renda e menor número de filhos. Estudos também apontam que maior escolaridade, renda e idade são influenciadores do apoio percebido (Castro et al., 1997; Crittenden, 1985; Griep et al., 2005; Hashima \& Amato, 1994; Leinonen et al., 2003), o que também poderia contribuir na explicação sobre a melhor percepção do apoio no Interior Leste.

Outro resultado encontrado foi na dimensão Apoio Material, na qual a Capital apresentou médias significativamente inferiores aos outros dois contextos. Esta dimensão do apoio refere-se à provisão de recursos práticos e ajuda material, como alguém para auxiliar nas tarefas diárias caso fique doente, para levá-la ao médico, etc. Umas das características da Capital é seu alto nível de urbanização, com elevados índices de escolaridade e renda. Apesar de apresentar também características mistas, a Capital (como outros ambientes de alta urbanização) apresenta maior valorização do desenvolvimento individual, com priorização das metas pessoais e com foco nas necessidades e direitos do indivíduo (Keller, et al., 2004; Keller, et al., 2005; Westphal et al., 2010). Com isso, nesses contextos é comum o individualismo e, em decorrência dele, uma diminuição da ajuda ao outro, especialmente no caso da ajuda material.

De modo diverso do verificado na diferença entre as outras dimensões, encontrada nos contextos do Interior, a dimensão de Apoio Material refere-se a uma questão mais concreta. Verificase que as questões envolvem a presença física ou material da ajuda e nos contextos mais interdependentes (Interior Leste e Oeste) essa dimensão não apresentou diferença, pois nesses modelos culturais é mais comum a união entre o grupo social.

Ainda com relação aos três contextos, após a realização da correlação de Pearson, diversas variáveis foram analisadas com a regressão múltipla, método stepwise. Essa análise permitiu que dois modelos estatisticamente significativos fossem apresentados. As correlações encontradas estão de acordo com os dados da literatura, especialmente com relação às variáveis sociodemográficas (Castro et al., 1997; Griep et al., 2005; Leinonem \& Solantaus, 2003) e o relacionamento com o parceiro (Cutrona et al., 1994; Dessen \& Braz, 2000; Montigny et al., 2006).

Os dois modelos explicativos trouxeram, assim como as correlações, coerência com a literatura pesquisada sobre o assunto. $\mathrm{O}$ modelo que melhor elucidou o apoio foi o segundo, com $10 \%$ da variância explicada. A renda já foi comentada anteriormente, contudo, sobre essa última variável, alguns detalhes ainda precisam ser enfatizados.

Compreende-se desse resultado que a "qualidade do ambiente familiar" atual foi uma variável importante para explicar as respostas das mães e que quanto melhor o ambiente familiar, mais elas percebem o apoio social. As questões que abarcavam a qualidade do ambiente familiar referiam-se predominantemente à relação conjugal, envolvendo a percepção sobre o relacionamento e as discussões. Quanto maior o índice se aproximava de cinco pontos, pior era o relacionamento do casal. Alguns estudos apontam que, especialmente durante a maternidade, a mãe tende a perceber o parceiro como fonte de apoio (Cutrona et al., 1994; Dessen \& Braz, 2000; Montigny et al., 2006) ou como a principal figura da qual recebe apoio (Vanderlinde et al., 2009). Contudo, nem sempre o parceiro pode estar disponível ou envolvido emocionalmente com a parentalidade, o que pode gerar conflitos conjugais e dificuldades no relacionamento, e se reflete na sensação de se sentir amada, segura e apoiada emocionalmente. $\mathrm{O}$ estudo realizado aponta nessa mesma direção, pois nele o parceiro é entendido como uma das principais figuras de apoio à mãe, e a qualidade do relacionamento entre ambos reflete-se diretamente na percepção do apoio social.

Ainda com referência à qualidade do ambiente familiar, essa variável envolvia o afeto parental. Como os estudos demonstram, quanto melhor o relacionamento do casal, mais afeto o pai apresenta para os filhos (Cutrona et al., 1994; Dessen \& Braz, 2000; Montigny et al., 2006). Assim, compreende-se que num relacionamento conjugal conturbado o pai demonstra menos afeto parental e a qualidade do ambiente familiar fica prejudicada e, por conseguinte, a percepção do apoio por parte da mãe.

A partir dessa discussão inicial foi possível responder algumas das hipóteses iniciais, que buscavam elucidar o modelo que melhor explicasse o apoio social nos contextos estudados, se havia relação do apoio social com a qualidade do ambiente familiar (que mostrou-se uma hipótese confirmada) e se os modelos culturais também poderiam explicar a diferença entre os contextos - o que também foi parcialmente confirmado. O próximo momento da discussão busca explanar sobre as especificidades de cada contexto estudado, direcionado para o objetivo de demonstrar diferenças e similaridades entre os mesmos.

Sobre as correlações de Pearson realizadas nos três contextos, pode-se indicar algumas similaridades entre elas. A Capital e o Interior Oeste apresentaram a "qualidade do ambiente familiar atual" como uma variável importante na percepção do apoio social. Essa tendência se repete no modelo de regressão múltipla discutido posteriormente. Essa presença, da mesma forma que elucidado anteriormente, enfatiza a importância da figura do parceiro como fonte de apoio para a mãe, especialmente durante a maternidade (Crittenden, 1985; Cutrona, 1994; Dessen \& Braz, 2000; Montigny et al., 2006).

A variável "escolaridade" demonstrou sua correlação também na Capital e no Interior Oeste. A escolarização já foi apontada em alguns estudos como correlacionada positivamente com o apoio social (Crittenden, 1985; Cutrona et al., 1994; Griep et al., 2005), relacionada principalmente com a melhor autoestima do indivíduo. A escolaridade teve uma correlação mais forte na Capital, aparecendo inclusive em seu modelo de regressão múltipla (a ser posteriormente melhor elucidado).

Outro ponto comum, agora entre todos os contextos, foi a conexão entre as dimensões da escala de apoio. Extrai-se dessa correlação que, apesar de diferenciadas, as dimensões da escala estão conectadas e, assim, o apoio social é um fenômeno único e percebido dessa forma. Apesar de características distintas entre o que é fornecido em cada dimensão e relação com a subjetividade da percepção do apoio, é comum que a rede que fornece o apoio emocional, afetivo e informacional também demonstre o suporte material (Berkman, 1984; Dessen \& Braz, 2000; Griep et al., 2005; Nelson, 2004). 
O Interior Leste apresentou uma peculiaridade nas correlações, demonstrando que a maior idade da mãe relacionase negativamente com o apoio percebido. Griep et al. (2005) apontam em seu estudo que pessoas mais velhas têm percepção menor do apoio social, principalmente pelo fato que o avançar da idade geram muitas modificações nas redes de suporte social e, portanto, no apoio social disponível. Apesar de nessa pesquisa as participantes terem na média uma idade jovem, percebe-se que quanto mais idade as mães apresentam, maior é o número de filhos. O Interior Oeste apresentou que quanto mais filhos, menos apoio a mãe percebeu. Como esses dois contextos do Interior se aproximam no modelo cultural, pode-se apontar uma possível relação entre a idade e a percepção do apoio. Contudo, esse resultado foi encontrado somente em um contexto, indicando mais uma distinção entre os locais estudados do que um resultado conclusivo da pesquisa.

Outra distinção encontrada entre os locais estudados foi a influência do número de filhos da percepção do apoio. $\mathrm{Na}$ Capital um número maior de filhos aumentou o apoio percebido, enquanto no Interior Oeste diminui. Essa distinção será melhor argumentada posteriormente, quando forem discutidos os modelos explicativos. Contudo, é importante arguir nesse momento que a parentalidade é uma fase de intensa transformação e estresse, em que o apoio social é uma forma não só de ajuda momentânea, mas também como preventivo da saúde mental dos pais e da criança (Bornstein et al., 2006; Montigny et al., 2006).

Sobre os modelos da regressão múltipla encontrados, é importante iniciar ressaltando a ausência do mesmo no contexto Interior Leste. A falta de um modelo justifica-se também pelas poucas e fracas correlações encontradas do apoio social com os dados sociodemográficos. As médias de apoio social desse contexto foram as que menos variaram, assim como os outros dados analisados. Com isso, não foi possível identificar as variáveis que melhor explicam o apoio social no Interior Leste.

Os outros dois contextos, Capital e Interior Oeste, apresentaram modelos muito parecidos para explicar o apoio social. Ambos apresentaram que a melhor qualidade do ambiente familiar aumenta a percepção do apoio social. O "número de filhos" apresentou-se contraditório nos modelos estudados, pois na Capital o maior número de filhos está relacionado a um aumento da percepção do apoio, enquanto no Interior Oeste sua presença diminui o apoio social percebido. A Capital também apresentou a "escolaridade" como variável independente estatisticamente significativa.

Sobre a presença da variável "qualidade do ambiente familiar atual" nos modelos de regressão da Capital e do Interior Oeste, discute-se da mesma forma que a presença no modelo de regressão explicativo dos três contextos conjuntamente. Como esta variável apresentava forte relação com o relacionamento com o companheiro e este é considerado uma das principais fontes de suporte para as mães no cuidado de seus filhos, o conflito marital demonstra estreita relação com a percepção do suporte parental (Cutrona et al., 1994; Dessen \& Braz, 2000; Montigny et al., 2006). É essencial ressaltar que a "qualidade do ambiente familiar atual" apresentou-se constante nesse estudo, confirmando sua forte relação com o apoio social percebido.
A presença em ambos os modelos da variável "número de filhos" pode ser compreendida com base no pressuposto de que a parentalidade é um momento de intenso estresse familiar, exigindo das famílias flexibilidade e segurança para lidar com seus filhos (Jennings et al., 1991; Koeske \& Koeske, 1990; Leinonen et al., 2003; Montigny et al., 2006). Em razão da especificidade desse momento, é esperado que a família procure mais o apoio, tanto material quanto emocional, o que pode gerar ou não a resposta dos parentes próximos e amigos a esse pedido de ajuda dos cuidadores. Mesmo que haja uma resposta da sua rede social, muitas vezes esse retorno não é considerado satisfatório pelos cuidadores, pois o número de tarefas e novas habilidades exigidas na parentalidade podem causar uma percepção negativa sobre sua rede de apoio social e a satisfação com esse suporte é essencial para o efeito benéfico da mesma (Montigny et al., 2006; Nelson, 2004; Sarason et al., 1983).

Assim, a presença da variável "número de filhos" indica que na Capital a mãe está percebendo o apoio e quanto mais ela identificar esse suporte social recebido, melhor tende a ser a capacidade de enfrentar as mudanças nesse momento (Cohen $\&$ Wills, 1985; Griep et al., 2005; Montigny et al., 2006), o que refletirá também nos cuidados com a criança (Bornstein et al., 2006; Crittenden, 1985; Dessen \& Braz, 2000; Langer et al., 1996).

Como descrito anteriormente, a escolaridade tem forte relação com o apoio percebido. Isso é explicado pelo fato de que pessoas com maior escolarização sentem-se mais seguras e autossuficientes, o que gera melhor autoestima e, consequentemente, uma percepção mais positiva de sua rede social (Crittenden, 1985; Cutrona et al., 1994; Griep et al., 2005). A escolaridade na Capital foi a mais alta, porém também foi o local com maior variação da resposta entre os três contextos, o que pode ter contribuído com a presença dessa variável somente no modelo da análise de regressão da Capital. Por outro lado, a "renda familiar mensal", que também foi assumida pelos autores do presente estudo como hipótese de predição do apoio percebido, apareceu correlacionada nos três contextos. Contudo, não foi constatada sua presença nos modelos explicativos, conforme era esperado. Essa ausência pode estar relacionada com a pouca variação da renda familiar dentro dos contextos, indicando um viés do estudo.

Os diversos resultados encontrados e discutidos na pesquisa voltam a enfatizar a importância do apoio social na saúde do ser humano, tanto nos momentos estressantes como no dia a dia. Já está bem estabelecida na literatura a importância do suporte social nas múltiplas dimensões da vida do indivíduo, especialmente aquelas ligadas à sua saúde física e mental (Andrade \& Vaitsman, 2002; Berkman, 1984; Berkman, 1995; Castro et al., 1997; Crittenden, 1985; Cutrona et al., 1994; Koeske \& Koeske, 1990; Matsukoya et al., 2002; Montigny et al., 2006).

A partir dos dados da literatura e da presente pesquisa, fica claro o papel do profissional em saúde mental de fortalecer as redes de apoio social, especialmente durante os momentos estressantes na vida do indivíduo. Da mesma forma, é possível apontar a importância da relação familiar, especialmente com o parceiro amoroso, na percepção do apoio. Como a pessoa sente 
o apoio é essencial para que os benefícios desse suporte sejam efetivos, então é importante que os estudos possam continuar, além de analisar quantitativamente, a investigar a percepção do apoio social e a satisfação da pessoa com a mesma.

As características culturais e sociodemográficas de cada contexto também apresentaram reflexo no modo como o apoio é percebido. O estudo de Westpahl et al. (2010) investigou esses mesmos contextos e encontrou diferenças referentes a questões de relação de grupo e cuidados parentais. O contexto Capital demonstrou características mais individualistas, com prevalência da valorização das dimensões de crenças e metas direcionadas ao desenvolvimento individual da criança; e o Interior Leste, a prevalência foi de características mistas nas metas e práticas e na proximidade com o grupo. Esses apontamentos foram discutidos também com relação aos dados sociodemográficos (escolaridade e renda) e características culturais do contexto.

No contexto Capital, a percepção das mães sobre Apoio Material foi menor, bem como os valores das médias nas outras dimensões da escala de apoio, demonstrando que esse suporte é menos percebido pelas mães de contextos mais urbanizados com relação ao Interior Leste. Fazendo um paralelo com o estudo de Westphal et al (2010), como o grupo social interage e as características sociodemográficas, são fatores que podem ser entendidos como relacionadas com a percepção do apoio social. Nesse sentido, contextos com menor proximidade do grupo (em termos de coesão) e maiores índices de urbanização podem refletir na menor percepção do apoio.

Nesse sentido, uma das contribuições desta pesquisa é salientar a necessidade de considerar variáveis relacionadas às diferenças regionais e culturais que podem ter influências na percepção do apoio e que possam ajudar os profissionais na avaliação da rede social relatada pelo indivíduo. Conhecer a comunidade e as formas de relação nas quais o sujeito está inserido favorecerá uma intervenção mais efetiva e uma ativação da rede com melhores benefícios.

A pesquisa, por ser de natureza quantitativa, teve algumas limitações. Primeiramente, não foram não foram investigadas as pessoas que estavam na rede social da mãe e também não foi identificada a principal figura de suporte. Além disso, a satisfação das participantes com a família próxima poderia indicar também resultados interessantes, pois os pais e parentes são também apontados como figuras essenciais ao apoio social (Cutrona et al., 1994; Dessen \& Braz, 2000; Montigny et al., 2006; Saranson et al., 1983; Vanderlinde et al., 2009).

A presente pesquisa, parte de um projeto nacional, propôsse a investigar diversos fenômenos e, em razão dos vários instrumentos existentes, o apoio social não pôde ser aprofundado. Com isso, justifica-se que o instrumento de apoio social utilizado nessa pesquisa deva ser novamente aplicado e aprofundado com uma abordagem também qualitativa e focada, assim como pesquisas que relacionam o apoio com outros fenômenos familiares, tais como práticas parentais.

\section{Referências}

Andrade, G. R. B., \& Vaitsman, J. (2002). Apoio social e redes: conectando solidariedade e saúde. Ciência \& Saúde Coletiva, 7(4), 925-934.
Azevedo, K. R., \& Arrais, A. R. (2006). O mito da mãe exclusiva e seu impacto na depressão pós-parto. Psicologia: Reflexão e Crítica, 19(2), 269-276. doi: http://dx.doi.org/10.1590/S0102-79722006000200013

Berkman, L. F. (1984). Assessing the physical health effects of social networks and social support. Annual Review of Public Health, 5, 413-432.

Berkman, L. F. (1995). The role of social relations in health promotion. Psychossomatic Medicine, 57, 245-254.

Bornstein, M. H., Putnick, D. L., Suwalsky, J. T. D., \& Gini, M. (2006). Maternal chronological age, prenatal history, social support, and parenting of infants. Child Development, 77(4), 875-892

Castro, R., Campero, L., \& Hernandez, B. (1997). La Investigación sobre apoyo social en salud: situación actual y nuevos desafíos. Revista de Saúde Pública, 31(4), 426-434. doi: http://dx.doi.org/10.1590/S0034-89101997000400012

Cohen, S., \& Wills, T. A. (1985). Stress, social support, and the buffering hypothesis. Psychological Bulletin, 98(2), 310-357.

Crittenden, P. M. (1985). Social networks, quality of child rearing, and child development. Child Development, 56, 1298-1313.

Cutrona, C. E., Cole, V., Colangelo, N., Assouline, S. G., \& Russel, D. W. (1994). Perceived parental social support and academic achievement: an attachment theory perspective. Journal of Personality and Social Psychology, 66(2), 369-378.

Dancey, C. P., \& Reidy, J. (2006). Estatística sem matemática para Psicologia. Porto Alegre: Artmed.

Dessen, M. A., \& Braz, M. P. (2000). Rede social de apoio durante transições familiares decorrentes do nascimento de filhos. Psicologia: Teoria e Pesquisa, 16(3), 221-231.

Goodwin, R., Cost, P., \& Adonu, J. (2004). Social support and its consequences: 'Positive' and 'deficiency' values and their implications for support and self-esteem. British Journal of Social Psychology, 43, 1-10.

Griep, R. H., Chor, D., Faerstein, E., Werneck, G. L., \& Lopes, C. S. (2005). Validade do constructo de escala de apoio social do Medical Outcomes Study adaptada para o português no Estudo Pró-Saúde. Cadernos de Saúde Pública, 21(3), 703-714. doi: http://dx.doi.org/10.1590/S0102-311X2005000300004

Hashima, P. Y., \& Amato, P. R. (1994). Poverty, social support, and parental behavior. Child Development, 65, 394-403.

Instituto Brasileiro de Geografia e Estatística. (2007). Cidades. Recuperado de http://www.ibge.gov.br.

Jennings, K. D., Stagg, V., \& Connors, R. E. (1991). Social networks and mothers' interactions with their preschool children. Child Development, 62, 966-978.

Keller, H., Borke, J., \& Yovsi, R. (2005). Cultural orientations and historical changes as predictors of parenting behaviour. International Journal of Behavioral Development, 29(3), 229-237. doi:10.1177/01650250544000017

Keller, H., Hentschel, E., Yovsi, R. D., Lamm, B., Abels, M., \& Haas, V. (2004). The psycho-linguistic embodiment of parental ethnotheories: A new avenue to understanding cultural processes in parental reasoning. Culture \& Psychology, 10(3), 293-330. doi: 10.1177/1354067X04042890

Koeske, G. F., \& Koeske, R. D. (1990). The buffering effect of social support on parental stress. American Journal Orthopsychiatric, 60(3), 440-451.

Langer, A., Farnot, U., Garcia, C., Barros, F., Victora, C., Belizan, J. M., \& Villar, J. (1996). The Latin American trial of psychosocial support during pregnancy: effects on mother's wellbeing an satisfaction. Social Science \& Medicine, 42(11), 1589-1597.

Leinonen, J. A., Solantus, T. S., \& Punamãki, R. J. (2003). Social support and the quality of parenting under economic pressure and workload in Finland: the role of family structure and parental gender. Journal of Family Psychology, 17(3), 409-418.

Matsukura, T. S., Maturano, E. M., \& Oishi, J. (2002). O questionário de suporte social (SSQ): estudos da adaptação para o português. Revista LatinoAmericana de Enfermagem, 10(5), 675-681.

Montigny, F., Lacharité, C., \& Amyot, E. (2006). The transition to fatherhood: the role of formal and informal support structures during the post-partum period. Contexto de Enfermagem, 15(4), 601-609. doi: http://dx.doi.org/10.1590/ 


\section{S0104-07072006000400008}

Nelson, T. N. (2004). The role of family values and perceived family social support in stress appraisal among black and white college students (Tese de Doutorado). Recuperado de http://etd.lib.fsu.edu/theses/available/etd11152004-172106/ . (etd-11152004-172106)

Sarason, I. G., Levine, H. M., Basham, R. B., \& Sarason, B. R. (1983). Assessing social suport: the social support questionnaire. Jounal of Personality and Social Psychology, 44(1), 127-139.
Sherbourne, C. D., \& Stewart, A. L. (1991). The MOS social support survey. Social Science \& Medicine, 32(6), 705-714.

Vanderlinde, L. F., Borba, G. A., \& Vieira, M. L. (2009). Importância da rede social de apoio para mães de crianças na primeira infância. Revista de Ciências Humanas, 43(2), 429-443.

Westphal, J. P., Vieira, V., Vieira, M. L., \& Prado, A. B. (2010). O que mães pensam sobre seus filhos em três regiões distintas do estado de Santa Catarina. Psicologia Argumento, 28(62), 235-246.

Viviane Vieira, graduada em Psicologia pela Universidade Federal de Santa Catarina, é mestranda em Psicologia pela Universidade de Santa Catarina. Endereço para correspondência: Rua Bocaiúva, 1659, apt 1001, Bairro Centro, Florianópolis-SC. CEP: 88015-530. Telefone: (48)9944-8112. E-mail: vivianepsicoufsc@gmail.com. Mauro Luis Vieira, doutor em Psicologia Experimental pela Universidade São Paulo, pós-doutor pela Dalhousie University, Canadá, é professor Associado da Universidade Federal de Santa Catarina. E-mail: maurolvieira@gmail.com Alessandra Bonassoli Prado, mestre em Psicologia pela Universidade Federal de Santa Catarina, é doutoranda em Psicologia Experimental na Universidade de São Paulo. Bolsista CNPq. E-mail: sanaprado@hotmail.com 